\title{
Telestroke: the promise and the challenge. Part one: growth and current practice
}

\author{
F Akbik, ${ }_{1}^{1}$ J A Hirsch, ${ }^{2,3}$ R V Chandra, ${ }^{4}$ D Frei, ${ }^{5}$ A B Patel, ${ }^{3,6}$ J D Rabinov, ${ }^{2,3}$ N Rost, \\ L H Schwamm, ${ }^{1}$ T M Leslie-Mazwi ${ }^{1,3}$
}

'Department of Stroke Neurology, Massachusetts General Hospital, Boston, Massachusetts, USA 2Department of Interventional Neuroradiology, Massachusetts General Hospital, Boston,

Massachusetts, USA ${ }^{3}$ Department of

Neuroendovascular

Massachusetts General Hospital, Boston, Massachusetts, USA ${ }^{4}$ Department of

Neuroendovascular, Monash University Hospital, Melbourne, Australia

${ }^{5}$ Department of Neurolnterventional Surgery, Radiology Imaging Associates/ RIA Neurovascular, Swedish Medical Center, Englewood, Colorado, USA

${ }^{6}$ Department of Neurosurgery, Massachusetts General Hospital, Boston, Massachusetts, USA

Correspondence to Dr T M Leslie-Mazwi, GRB 2-241, 55 Fruit Street, Boston, MA 02114, USA; tlesliemazwi@mgh.harvard.edu

Received 14 February 2016 Accepted 20 February 2016 Published Online First 16 March 2016

\section{SLinked}

http://dx.doi.org/10.1136/ neurintsurg-2016-012340

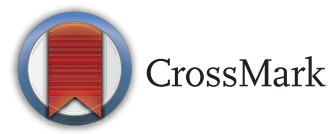

To cite: Akbik F, Hirsch JA Chandra RV, et al. J Neurolntervent Surg 2017; 9:357-360.

\section{ABSTRACT}

Acute ischemic stroke remains a major public health concern, with low national treatment rates for the condition, demonstrating a disconnection between the evidence of treatment benefit and delivery of this treatment. Intravenous thrombolysis and endovascular thrombectomy are both strongly evidence supported and exquisitely time sensitive therapies. The mismatch between the distribution and incidence of stroke presentations and the availability of specialist care significantly affects access to care. Telestroke, the use of telemedicine for stroke, aims to surmount this hurdle by distributing stroke expertise more effectively, through video consultation with and examination of patients in locations removed from specialist care. This is the first of a detailed two part review, and explores the growth and current practice of telestroke, including the specific role it plays in the assessment and management of patients after emergent large vessel occlusion.

\section{INTRODUCTION}

Despite significant advances in primary prevention, acute ischemic stroke (AIS) remains a major public health burden. AIS is the fourth leading cause of mortality in the USA and the top cause of serious long term disability. ${ }^{1}{ }^{2}$ Acute disease modifying treatment is founded on the recanalization hypothesis; recanalization of the occluded cerebral artery results in penumbral salvage, if accomplished early enough.

Prior to 1996, no Food and Drug Administration approved therapy directed to this goal existed, and acute management was supportive. The utilization of tissue plasminogen activator (tPA) in AIS revolutionized modern stroke therapy, ${ }^{3}{ }^{4}$ and in 1996 became the first directed therapy for AIS. Management of AIS has since rapidly evolved, both in application and understanding. The critical time dependence of tPA effect is well recognized, ${ }^{5}$ but in 2009 the window for intravenous (IV) tPA was extended, ${ }^{67}$ and dedicated stroke units ${ }^{89}$ are now recognized to optimize stroke management and outcomes. Recently, endovascular therapies have entered the mainstream, providing an effective treatment for patients with proximal large vessel occlusions, with improved recanalization, functional outcomes, and survival, ${ }^{10-12}$ a population of patients who may otherwise not benefit from tPA.

Despite these advances, a mere $5 \%$ of all ischemic stroke patients are treated with thrombolysis in the USA and Europe, ${ }^{13}{ }^{14}$ highlighting the need to optimize stroke care systems. Challenges exist at several levels. Public awareness of stroke symptomatology and urgency remains limited, ${ }^{15}{ }^{16}$ and the overwhelming majority of eligible patients present outside of the therapeutic window for IV $\mathrm{tPA}^{17}$ Once a patient presents to medical attention, significant hurdles exist with treatment delivery. The mismatch between AIS incidence and subspecialty availability greatly affects access to care. Nationally, there is a prominent shortage of vascular neurologists, with only 1100 subspecialists despite an incidence of 800000 strokes per year. ${ }^{18}$ Nearly half of all hospitals in the USA do not have a neurologist on staff, and only $55 \%$ of all Americans live within 60 miles of a primary stroke center. ${ }^{19}$ The discrepancy is most pronounced in the 'stroke belt' (southeastern USA), which has both the greatest incidence of AIS and lowest density of neurologists. ${ }^{20-22}$ Even when neurologists live in the area, the increasing shift towards outpatient practices has decreased neurology coverage of local emergency departments. ${ }^{23-25}$

In the absence of a local specialist, management of AIS often is directed by local emergency medicine physicians who may be less comfortable administering tPA. Even under ideal conditions and clear indications, $40 \%$ of surveyed emergency medicine physicians would be unlikely to administer tPA. ${ }^{26}$ The most commonly cited concerns are inexperience with tPA, concerns for intracranial hemorrhage (ICH), and medicolegal liability ${ }^{26} 27$ (even though malpractice claims for not treating IV tPA eligible patients far outweigh those when IV tPA was administered ${ }^{28}$.

In particular, the mixed data correlating protocol violations with increased rates of $\mathrm{ICH},{ }^{29-31}$ along with the concern for increased rates of observed ICH in routine practice compared with the National Institute of Neurological Disorders and Stroke (NINDS) trial, ${ }^{3} 2930$ have limited the willingness of non-neurologists to thrombolyse patients despite more recent data demonstrating parity between routine practice and clinical trial outcomes following appropriate training. ${ }^{32}$

Consequently, thrombolytics are grossly underutilized in rural and underserved communities. In a review of Medicare billing records of 4750 hospitals and nearly 500000 cases of AIS over a 2 year period, $62 \%$ of surveyed hospitals never administered tPA. $^{33}$ Consistent with the lower specialist density, ${ }^{20}$ patients in the midwest and southeast were least likely to receive tPA. ${ }^{33}$ Even within a given state, thrombolysis rates were almost 10 times lower in remote communities than in urban settings. ${ }^{34}$ 


\section{GENESIS AND GROWTH OF TELESTROKE}

Telestroke, ${ }^{35}$ the use of telemedicine for AIS care, is a modern strategy to overcome the practical limitations of stroke care delivery.

AIS is uniquely suited to telemedicine, for the following reasons.

1. A wide geographic and population distribution of disease.

2. Clearly visible clinical findings, often readily identifiable on video.

3. A narrow therapeutic window.

4. An existing, proven therapy that is predominantly IV and therefore can be administered in any facility with basic infrastructure.

5. Limited specialist availability.

With the aid of local hospital staff, a remote neurologist is able to use modern telecommunications networks to conduct a rapid clinical assessment, irrespective of locale, and based on this evaluation make treatment recommendations for the local clinician team.

Telestroke was initially pioneered in the USA, where a Boston based stroke center partnered with a hospital in Martha's Vineyard to demonstrate that remote neurologic assessment was reliable and increased the rates of thrombolysis. ${ }^{36-38}$ Based on this proof of principle, a German effort led to the creation of the first telestroke network in which rural facilities in Swabia (TESS network) and Bavaria (TEMPiS network) were connected to central hubs with extended stroke care capabilities. ${ }^{39} 40$ These pilot studies demonstrated the feasibility of a large scale telestroke network in facilitating remote thrombolysis. Telestroke models have since been widely adopted ${ }^{41}$ and endorsed by the American Heart Association (AHA). ${ }^{42}$

\section{The rise of telestroke networks}

Telestroke has quickly spread throughout the USA, with at least 56 networks in 27 states linking a stroke center with local hospitals that lack around the clock stroke expertise. ${ }^{41}$ The particulars of each arrangement have led to network based experimentation as individual programs have explored a range of services and delivery models.

A remote neurologist can offer a range of services through a telemedicine platform, through either video or telephone contact. A variety of different models exist, but evidence suggests telestroke (or more broadly telemedicine), with audio and video, is superior to telephone consultation alone. ${ }^{43}$ Practice models include the following:

1. Teleconsultation: a remote neurologist offers only advisory services, ${ }^{44}$ and the referring hospital maintains responsibility and liability for the patient.

2. Telethrombolysis: a remote neurologist decides on thrombolysis and is responsible to varying degrees for the patient's course, typically in the context of a pre-existing agreement between hospitals.

3. Integrated telehealth system: exchange of patients, information, and therapeutic suggestions between centers involved in acute stroke care, often with disseminated responsibilities and no clearly dominant hub.

These services are offered through a variety of stroke networks, ranging from individual arrangements between hospitals to multilayered referral networks. The earliest American model was a one to one partnership, where a remote island hospital contracted with a tertiary referral center for around the clock stroke coverage. ${ }^{45}$ Building on this, the early German pilot studies pioneered the classic spoke and hub model, ${ }^{39} 40$ where a central referral center partners with multiple local facilities to provide around the clock stroke coverage. This is now the dominant network model. The spoke and hub model has since become more nuanced, as rural hospitals partner with local primary stroke centers (sub-hubs) who are themselves partnered with a comprehensive stroke center (hub). The sub-hub provides routine stroke care services, but patients requiring subspecialty care or intervention are transferred to the hub, which is usually a comprehensive stroke center. More recently, an English telestroke network pioneered a horizontal telestroke model in rural hospitals that have local neurologists but lack both around the clock coverage and access to a tertiary referral center. ${ }^{46}$ Through a reciprocal agreement, hospitals take turns providing after hours stroke coverage, and patients continue stroke therapy the following day at the local hospital. ${ }^{46}$ By leveraging the speed and reach of telecommunication, all delivery networks are able to extend specialty and subspecialty care to broad geographic regions using preexisting resources.

Once a telestroke service identifies an AIS case, patients can either be transported to a referral hospital or kept at the local hospital. In the 'drip and ship' model, a remote neurologist decides to initiate thrombolysis and transfer the patient for admission to their service. The patient immediately receives tPA and is then transferred to the hub or sub-hub facility. This most frequently occurs with small local hospitals without the staff or capacity to provide stroke care. Alternatively, many remote hospitals have stroke care capacity, but lack around the clock stroke coverage. In these circumstances, teleconsultations for mild to moderate strokes may result in 'drip and keep,' where tPA is administered and patients remain at the local hospital with local neurology coverage the following morning. A remote specialist can also continue to advise regarding a patient's care through a telemedicine enabled local hospital unit. Patients with severe strokes, requiring endovascular intervention, surgical intervention (e.g., hemicraniectomy), and critical care should be transferred to the tertiary center in the absence of local expertise or facilities. In all models, network hospitals have specific agreements and protocols to transfer patients with stroke or thrombolysis complications.

\section{TELESTROKE IN CLINICAL PRACTICE}

With the increasing adoption of telestroke, a growing body of evidence has evaluated the technical feasibility, diagnostic fidelity, safety, and efficacy of telestroke in AIS management.

\section{Telestroke technology}

Telemedicine is dependent on the technology that makes it possible. Initial experiences with video teleconferencing were limited by the transmission rates of integrated services digital network (ISDN) or dedicated local area network (LAN) connections. ${ }^{38}$ Rare but notable interruptions complicated initial pilot studies. ${ }^{37}$ The proliferation of broadband and cellular access, combined with high resolution video capability, has since facilitated high resolution, low latency audiovisual connections. ${ }^{47}$ Initial videoconferencing technology required the remote neurologist to be present at a fixed workstation, ${ }^{38}$ but technical advances have since led to laptop ${ }^{47} 48$ and even smartphone based videoconferencing. ${ }^{49-51}$ Now that telemedicine capability is routine with a low barrier to entry, the principle concern has shifted away from technical to practical limitations over the implementation and utility of remote assessment.

\section{Telestroke and diagnostic accuracy}

Initial experimentation with telestroke has consistently demonstrated that video teleconference facilitates rapid and accurate 
remote diagnosis. In an initial pilot study done in the non-acute setting, remote application of the National Institutes of Health Stroke Scale (NIHSS) was assessed in tPA ineligible AIS patients. ${ }^{38}$ Nurse assisted remote evaluation by a neurologist was comparable with that of a bedside neurologist, with a discordance rate similar to that observed between two bedside examiners. $^{52} 53$ Nonetheless, while remote diagnostic assessment is rapid, it is slower than a bedside assessment $(9.7$ vs 6.5 min, respectively). ${ }^{38}$ The reliability of remote NIHSS assessment has since been replicated in the acute setting, ${ }^{54} 55$ demonstrating the fidelity of telestroke assessment in AIS. In an attempt to further optimize remote diagnosis, video teleconference and telephone consultation were directly compared in the prospective Stroke Team Remote Evaluation Using a Digital Observation Camera (STRokE DOC) trial. ${ }^{43}$ Using a mobile, laptop based system, video teleconference led to a correct diagnosis and thrombolysis in over $97 \%$ of cases, with an OR for a correct diagnosis of 10.9 compared with telephone consultations. Based on initial experiences with telestroke, the AHA now endorses remote clinical and radiographic assessments of patients in underserved medical settings as safe and reliable (class IA). ${ }^{42}$

\section{Safety of remote thrombolysis}

The emphasis on accuracy of remote diagnosis is essential because of the safety concerns of remote thrombolysis. Given the potential for tPA induced $\mathrm{ICH}$, the risk is that inappropriate thrombolysis could precipitate an iatrogenic ICH. The most likely reasons for inappropriate thrombolysis are protocol violations and misdiagnosis of a stroke mimic. Stroke mimics are equally common among local and referral hospitals, ${ }^{56} 57$ and telestroke is effective in identifying cases of stroke mimics. ${ }^{56}$ Even when stroke mimics are inappropriately thrombolysed, ICH is extremely rare and less likely to occur than in thrombolysed AIS patients. ${ }^{58}$ Alternatively, protocol violations could result in either inappropriate thrombolysis or inadequate perithrombolysis management, increasing the risk of $\mathrm{ICH}^{29-31}$ While protocol violations are not uncommon in community practice, ${ }^{29}$ violations are rare at local hospitals in a telestroke network ${ }^{36} 4043$ and are comparable with rates observed in the network hub. ${ }^{40}$ This is likely due to the fact the same pool of hub based neurologists are deciding on thrombolysis for the entire telestroke network. Ultimately, the safety of telestroke is measured by rates of $\mathrm{ICH}$, irrespective of the appropriate diagnosis or management. Multiple telestroke networks have now demonstrated that remote thrombolysis does not increase the risk of ICH compared with either in-person thrombolysis at a referral center or published trial data. ${ }^{43}{ }^{59-62}$ Remote thrombolysis is therefore both accurate and safe.

\section{Telestroke and thrombolysis rates}

The increasing use of telestroke has created a body of data to test the assumption that telestroke would increase thrombolysis rates (and by extension improve clinical outcomes). ${ }^{63}$ Universally, implementation of a telestroke network is associated with a significant increase in thrombolysis in remote or underserved hospitals. $^{40} 43 \quad 5961 \quad 62 \quad 64-67$ Consider rural Georgia, where as recently as 2002, tPA was simply not administered to patients with AIS due to the lack of available neurologists. After a telestroke network was implemented the following year, tPA utilization rates immediately increased to $16 \%$ in networked hospitals. ${ }^{54}{ }^{68}$ Similarly, in rural Bavaria, Germany, tPA utilization increased nearly 10 -fold after the implementation of a telestroke network while out of network tPA utilization lagged behind. ${ }^{67}$ Critically, remote hospitals in a telestroke network use tPA at equivalent rates to referral centers, demonstrating a tractable response to the rural penalty in AIS. ${ }^{54} 6669$

\section{Telestroke and stroke outcomes}

Telestroke improves stroke outcomes. This is the most important component of this therapeutic modality. The increased use of tPA with telestroke has led to direct comparisons of clinical outcomes in networked and out of network hospitals. In a prospective study comparing 3 month outcomes after an AIS in 3122 cases, the composite outcome of death, institutionalization, or disability was significantly less likely in telestroke hospitals than comparable out of network hospitals (44\% and 54\%, respectively). ${ }^{60}$ In a multivariate regression analysis, presentation to a telestroke hospital was independently associated with a reduced risk of a poor outcome. ${ }^{60}$ The benefit of telestroke also extends beyond thrombolysis, as demonstrated in a separate German telestroke network. ${ }^{39}$ Remote consultation led to higher rates of diagnostic and therapeutic interventions in cases of AIS, with at least $75 \%$ of consultations resulting in a meaningful change in management. This is consistent with a previous observation from the Veterans Administration Acute Stroke study where neurologist management of AIS was associated with both increased testing and improved clinical outcomes, including reduction in death and disability. ${ }^{70}$

Consequently, the availability of telestroke not only improved clinical outcomes relative to other remote hospitals, but it has also closed the rural gap in outcomes compared with large referral centers. Multiple telestroke networks throughout Europe and the USA have reported comparable rates of disability and mortality between stroke centers and telestroke hospitals. ${ }^{59-6164697172}$ In a retrospective review of 296 cases of thrombolysis in a Boston based telestroke network, there was no difference in symptomatic ICH, mortality, discharge disposition, and long term functional outcomes between patients thrombolysed remotely in small community hospitals or at a regional stroke center. ${ }^{59}$ Notably, all thrombolysed patients were transferred in this network, but improved clinical outcomes do not require patient transfer. This was demonstrated in a Finnish telestroke network that was based on a drip and keep delivery model. ${ }^{61}$ Even when patients were kept at small remote hospitals, there was no difference in mortality or functional outcome. Telestroke therefore improves clinical outcomes across multiple delivery models, providing subspecialty care to underserved communities. Effectively, the functional characteristics of the specialist center are brought to the local hospital emergency room.

\section{Telestroke and endovascular therapy}

It is now clear from recent data that in selected patients with emergent large vessel occlusions, endovascular management improves functional outcomes and mortality. ${ }^{10-12} 73$ Similar to IV thrombolysis, remote and underserved communities have limited access to endovascular therapy, ${ }^{74}$ but limited access is also a concern in urban regions with eccentric service provision. Telestroke provides an opportunity to extend the reach of endovascular therapy evaluation to patients who would otherwise do poorly.

Accurate identification of potential embolectomy candidates is the primary focus, most readily through accurate video assessment of NIHSS. Any patient with an NIHSS score greater than 6 is a potential endovascular candidate, ${ }^{75}$ but higher NIHSS thresholds more accurately predict proximal vessel occlusions. ${ }^{76}$ Additionally, if vascular imaging is obtained at the referral 
source, central review of images can be performed remotely to determine anatomic candidacy for therapy (level of occlusion, tortuosity, collateral vasculature, etc). Telestroke in this fashion provides effective triage of patients likely to benefit from more invasive treatment options.

Beyond appropriate identification and triage of endovascular candidates, telestroke availability may potentially facilitate prompt transfer, potentially overcoming time delays that represent a major barrier to endovascular therapy ${ }^{77} 78$ by allowing preparation for the endovascular case to occur while the patient is in transit. In addition, telestroke based consent from family members for mechanical thrombectomy can be accomplished through a video interface, allowing a detailed discussion of the planned intervention. This has been demonstrated in a Spanish telestroke network where remote assessment facilitated prompt thrombolysis and transfer for endovascular management of AIS. ${ }^{79}$ In this 'drip, ship, and retrieve' model, informed consent was obtained remotely and the angiography suite was prepared while the patient was en route, facilitating faster groin puncture times in telestroke versus out of network transfers. Three months post-infarct, functional outcomes were comparable between patients initially treated at the referral center or via telestroke, but significantly worse in patients in out of network facilities.

Telestroke is therefore a strategy with the potential to bridge the geographic gap in access to both pharmacologic and mechanical recanalization (box 1).

Box 1 Role of telestroke for potential endovascular stroke treatment candidates and for endovascular practice

Clinical identification of candidacy for endovascular intervention

- Presence/nature of deficit

- Magnitude of deficit (NIHSS)

- Clarification/determination of symptom onset timing

Radiological identification of candidacy for endovascular intervention

- Exclusion of hemorrhage

- Presence of ELVO (hyperdense vessel on NCHCT or vessel occlusion on (TA)

- Adequacy of collaterals

- Volume of established core

- Arterial access considerations (tortuosity, etc)

Facilitated patient transfer decisions

- Helicopter vs road

Advance procedural preparation

- Angiographic suite

- Equipment preparation

- Anesthesia mobilization

Video based procedural consent from family

Feedback/follow-up to referring hospital

- Logistics of transfer

- Patient evaluation and course

- Patient outcome

- Quality improvement efforts

Expanded general endovascular referrals

- Hemorrhagic pathology

- Non-acute ischemic pathology

CTA, CT angiogram; ELVO, emergent large vessel occlusion;

NCHCT, non-contrast head CT; NIHSS, National Institutes of Health Stroke Scale.
Impact of telestroke beyond acute stroke treatment

Beyond the acute phase, telestroke has led to improvements in a range of overall AIS management at network hospitals. Critically, these interventions benefit both thrombolysed and untreated patients. For instance, a German telestroke network implemented local stroke units in all networked hospitals, providing standardized treatment protocols and medical education to the local staff on optimal stroke management and secondary prevention. ${ }^{80}$ Compared with out of network hospitals, telestroke hospitals had a decreased composite risk of death, disability, or institutionalization. ${ }^{80}$ Similarly, evidence based guideline implementation interventions in the USA, such as the Get With the Guidelines-Stroke program, are likely more accessible in telestroke hospitals than non-telestroke centers. In the subacute setting, telestroke has also been used to remotely provide transient ischemic attack management and secondary prevention. ${ }^{81} 82$ By harnessing the reach of telecommunication, telestroke has the potential to provide both preventative and acute management to underserved communities.

\section{Summary}

Despite the advent of effective pharmacologic and mechanical treatments, healthcare delivery for AIS is limited by the current allocation of resources. Telestroke was developed as a response to geographic disparities, leveraging modern telecommunication technology to extend existing resources into underserved communities. Now, with more than a decade of experience, the safety, efficacy, and improved long term outcomes demonstrated by telestroke have firmly established it as a durable healthcare delivery model. With the advent of data strongly supporting the use of endovascular therapy in stroke, the relevance of telestroke has never been greater. In the second part of this two part review, we will discuss the challenges telestroke faces for wider adoption.

Contributors TML-M, JAH, DF, and FA designed the work and the initial draft. $R V C, N R, A B P, J D R$, and LHS revised the article in collaboration with all of the listed authors.

Competing interests JAH declares that he holds shares in Intratech Medical, unrelated to the present work.

Provenance and peer review Commissioned; internally peer reviewed.

\section{REFERENCES}

1 Roger VL, Go AS, Lloyd-Jones DM, et al. American Heart Association Statistics Committee and Stroke Statistics Subcommittee, Heart disease and stroke statistics2012 update: a report from the American Heart Association. Circulation 2012;125: e2-220.

2 Go AS, Mozaffarian D, Roger VL, et al. Heart disease and stroke statistics-2013 update: a report from the American Heart Association. Circulation 2013;127: e6-245.

3 The National Institute of Neurological Disorders and Stroke rt-PA Stroke Study Group. Tissue plasminogen activator for acute ischemic stroke. $N$ Engl J Med 1995;333:1581-8.

4 Brott TG, Haley EC, Levy DE, et al. Urgent therapy for stroke. Part I. Pilot study of tissue plasminogen activator administered within 90 minutes. Stroke 1992;23:632-40.

5 Lees KR, Bluhmki E, von Kummer $\mathrm{R}$, et al. Time to treatment with intravenous alteplase and outcome in stroke: an updated pooled analysis of ECASS, ATLANTIS, NINDS, and EPITHET trials. Lancet 2010;375:1695-703.

6 Hacke W, Kaste M, Bluhmki E, et al. Thrombolysis with alteplase 3 to 4.5 hours after acute ischemic stroke. N Engl J Med 2008;359:1317-29.

7 Del Zoppo GJ, Saver JL, Jauch EC, et al. Expansion of the time window for treatment of acute ischemic stroke with intravenous tissue plasminogen activator: a science advisory from the American Heart Association/American Stroke Association. Stroke 2009;40:2945-8.

8 Jørgensen $\mathrm{HS}$, Nakayama H, Raaschou HO, et al. The effect of a stroke unit: reductions in mortality, discharge rate to nursing home, length of hospital stay, and cost: a community-based study. Stroke 1995;26:1178-82. 
9 Indredavik B, Bakke $F$, Solberg $R$, et al. Benefit of a stroke unit: a randomized controlled trial. Stroke 1991;22:1026-31.

10 Berkhemer OA, Fransen PS, Beumer D, et al. A randomized trial of intraarterial treatment for acute ischemic stroke. N Engl J Med 2015:372:11-20.

11 Goyal M, Demchuk AM, Menon BK, et al. Randomized assessment of rapid endovascular treatment of ischemic stroke. N Engl J Med 2015:372:1019-30.

12 Campbell BC, Mitchell PJ, Kleinig TJ, et al. Endovascular therapy for ischemic stroke with perfusion-imaging selection. N Engl J Med 2015:372:1009-18.

13 Adeoye 0 , Hornung $R$, Khatri $P$, et al. Recombinant tissue-type plasminogen activator use for ischemic stroke in the United States: a doubling of treatment rates over the course of 5 years. Stroke 2011;42:1952-5.

14 Leys $D$, Ringelstein EB, Kaste $M$, et al. Facilities available in European hospitals treating stroke patients. Stroke 2007;38:2985-91.

15 Schneider AT, Pancioli AM, Khoury JC, et al. Trends in community knowledge of the warning signs and risk factors for stroke. JAMA 2003:289:343-6.

16 Pancioli AM, Broderick J, Kothari R, et al. Public perception of stroke warning signs and knowledge of potential risk factors. JAMA 1998;279:1288-92.

17 Kleindorfer D, Kissela B, Schneider A, et al. Eligibility for recombinant tissue plasminogen activator in acute ischemic stroke: a population-based study. Stroke 2004;35:e27-9.

18 Leira EC, Kaskie B, Froehler MT, et al. The growing shortage of vascular neurologists in the era of health reform: planning is brain! Stroke 2013;44:822-7.

19 Albright KC, Branas CC, Meyer BC, et al. Access: acute cerebrovascular care in emergency stroke systems. Arch Neurol 2010;67:1210-18.

20 Dall TM, Storm MV, Chakrabarti R, et al. Supply and demand analysis of the current and future US neurology workforce. Neurology 2013;81:470-8.

21 Gillum RF, Ingram DD. Relation between residence in the southeast region of the United States and stroke incidence: the NHANES I epidemiologic followup study. Am J Epidemiol 1996;144:665-73.

22 Adornato BT, Drogan 0, Thoresen P, et al. The practice of neurology, 2000-2010: report of the AAN member research subcommittee. Neurology 2011;77:1921-8.

23 McConnell KJ, Johnson LA, Arab N, et al. The on-call crisis: a statewide assessment of the costs of providing on-call specialist coverage. Ann Emerg Med 2007:49:727-33.e18

24 Rudkin SE, Langdorf MI, Oman JA, et al. The worsening of ED on-call coverage in California: 6-year trend. Am J Emerg Med 2009;27:785-91.

25 Freeman WD, Vatz KA, Griggs RC, et al. The workforce task force report: clinical implications for neurology. Neurology 2013;81:479-86.

26 Brown DL, Barsan WG, Lisabeth LD, et al. Survey of emergency physicians about recombinant tissue plasminogen activator for acute ischemic stroke. Ann Emerg Med 2005:46:56-60.

27 Liang BA, Zivin JA. Empirical characteristics of litigation involving tissue plasminogen activator and ischemic stroke. Ann Emerg Med 2008;52:160-4.

28 Bhatt A, Safdar A, Chaudhari D, et al. Medicolegal considerations with intravenous tissue plasminogen activator in stroke: a systematic review. Stroke Res Treat 2013:2013:562564

29 Katzan IL, Furlan AJ, Lloyd LE, et al. Use of tissue-type plasminogen activator for acute ischemic stroke: the cleveland area experience. JAMA 2000;283:1151-8.

30 Bravata DM, Kim N, Concato J, et al. Thrombolysis for acute stroke in routine clinical practice. Arch Intern Med 2002;162:1994-2001.

31 Lopez-Yunez AM, Bruno A, Williams LS, et al. Protocol violations in community-based rtPA stroke treatment are associated with symptomatic intracerebral hemorrhage. Stroke 2001:32:12-16.

32 Katzan IL, Hammer MD, Furlan AJ, et al. Quality improvement and tissue-type plasminogen activator for acute ischemic stroke: a Cleveland update. Stroke 2003;34:799-800.

33 Kleindorfer D, Xu Y, Moomaw CJ, et al. US geographic distribution of rt-PA utilization by hospital for acute ischemic stroke. Stroke 2009;40:3580-4.

34 Miley ML, Demaerschalk BM, Olmstead NL, et al. The state of emergency stroke resources and care in rural Arizona: a platform for telemedicine. Telemed J E Health 2009:15:691-9.

35 Levine SR, Gorman M. "Telestroke": the application of telemedicine for stroke. Stroke 1999;30:464-9.

36 Schwamm LH, Rosenthal ES, Hirshberg A, et al. Virtual telestroke support for the emergency department evaluation of acute stroke. Acad Emerg Med 2004:11:1193-7.

37 Lamonte MP, Bahouth MN, Hu P, et al. Telemedicine for acute stroke: triumphs and pitfalls. Stroke 2003:34:725-8.

38 Shafqat S, Kvedar JC, Guanci MM, et al. Role for telemedicine in acute stroke: feasibility and reliability of remote administration of the NIH stroke scale. Stroke 1999;30:2141-5.

39 Wiborg A, Widder B, Telemedicine in stroke in Swabia Project. Teleneurology to improve stroke care in rural areas: the Telemedicine in Stroke in Swabia (TESS) Project. Stroke 2003;34:2951-6.

40 Audebert HJ, Kukla C, Clarmann Von Claranau S, et al. Telemedicine for safe and extended use of thrombolysis in stroke: the Telemedic Pilot Project for Integrative Stroke Care (TEMPiS) in Bavaria. Stroke 2005:36:287-91.
41 Silva GS, Farrell S, Shandra E, et al. The status of telestroke in the United States: a survey of currently active stroke telemedicine programs. Stroke 2012;43:2078-85.

42 Schwamm LH, Holloway RG, Amarenco P, et al. A review of the evidence for the use of telemedicine within stroke systems of care: a scientific statement from the American Heart Association/American Stroke Association. Stroke 2009:40:2616-34.

43 Meyer BC, Raman R, Hemmen T, et al. Efficacy of site-independent telemedicine in the STRokE DOC trial: a randomised, blinded, prospective study. Lancet Neurol 2008; 7:787-95.

44 Bowry R, Parker S, Rajan SS, et al. Benefits of stroke treatment using a mobile stroke unit compared with standard management: the BEST-MSU study run-in phase. Stroke 2015;46:3370-4.

45 Schwamm LH, Audebert HJ, Amarenco P, et al. Recommendations for the implementation of telemedicine within stroke systems of care: a policy statement from the American Heart Association. Stroke 2009:40:2635-60.

46 Agarwal S, Day DJ, Sibson L, et al. Thrombolysis delivery by a regional telestroke network - experience from the UK National Health Service. J Am Heart Assoc 2014;3:e000408. doi:10.1161/JAHA.113.000408.

47 Meyer BC, Lyden PD, Al-Khoury L, et al. Prospective reliability of the STRokE DOC wireless/site independent telemedicine system. Neurology 2005;64:1058-60.

48 Audebert $\mathrm{HJ}$, Boy $\mathrm{S}$, Jankovits $\mathrm{R}$, et al. Is mobile teleconsulting equivalent to hospital-based telestroke services? Stroke 2008;39:3427-30.

49 Demaerschalk BM, Vegunta S, Vargas BB, et al. Reliability of real-time video smartphone for assessing National Institutes of Health Stroke Scale scores in acute stroke patients. Stroke 2012;43:3271-7.

50 Anderson ER, Smith $\mathrm{B}$, Ido $\mathrm{M}$, et al. Remote assessment of stroke using the iPhone 4. J Stroke Cerebrovasc Dis 2013;22:340-4.

51 Demaerschalk BM, Vargas JE, Channer DD, et al. Smartphone teleradiology application is successfully incorporated into a telestroke network environment. Stroke 2012:43:3098-101.

52 Brott T, Adams HP, Olinger CP, et al. Measurements of acute cerebral infarction: a clinical examination scale. Stroke 1989:20:864-70.

53 Goldstein LB, Bertels C, Davis JN. Interrater reliability of the NIH stroke scale. Arch Neurol 1989;46:660-2.

54 Wang S, Lee SB, Pardue $C$, et al. Remote evaluation of acute ischemic stroke: reliability of National Institutes of Health Stroke Scale via telestroke. Stroke 2003:34:e188-91.

55 Handschu R, Littmann R, Reulbach $U$, et al. Telemedicine in emergency evaluation of acute stroke: interrater agreement in remote video examination with a novel multimedia system. Stroke 2003;34:2842-6.

56 Ali SF, Viswanathan A, Singhal AB, et al. The TeleStroke mimic (TM) score: a prediction rule for identifying stroke mimics evaluated in a Telestroke Network. J Am Heart Assoc 2014;3.

57 Harbison J, Hossain 0, Jenkinson D, et al. Diagnostic accuracy of stroke referrals from primary care, emergency room physicians, and ambulance staff using the face arm speech test. Stroke 2003:34:71-6.

58 Chernyshev OY, Martin-Schild S, Albright KC, et al. Safety of tPA in stroke mimics and neuroimaging-negative cerebral ischemia. Neurology 2010;74:1340-5.

59 Pervez MA, Silva G, Masrur S, et al. Remote supervision of IV-tPA for acute ischemic stroke by telemedicine or telephone before transfer to a regional stroke center is feasible and safe. Stroke 2010;41:e18-24.

60 Audebert HJ, Schenkel J, Heuschmann PU, et al. Effects of the implementation of a telemedical stroke network: the Telemedic Pilot Project for Integrative Stroke Care (TEMPiS) in Bavaria, Germany. Lancet Neurol 2006;5:742-8.

61 Sairanen $T$, Soinila S, Nikkanen M, et al. Two years of Finnish Telestroke: thrombolysis at spokes equal to that at the hub. Neurology 2011;76:1145-52.

62 Lazaridis C, Desantis SM, Jauch EC, et al. Telestroke in South Carolina. J Stroke Cerebrovasc Dis 2013;22:946-50.

63 Müller-Barna P, Schwamm LH, Haberl RL. Telestroke increases use of acute stroke therapy. Curr Opin Neurol 2012;25:5-10.

64 Schwab S, Vatankhah B, Kukla C, et al. Long-term outcome after thrombolysis in telemedical stroke care. Neurology 2007;69:898-903.

65 Choi JY, Porche NA, Albright KC, et al. Using telemedicine to facilitate thrombolytic therapy for patients with acute stroke. Jt Comm J Qual Patient Saf 2006;32:199-205.

66 Amorim E, Shih MM, Koehler SA, et al. Impact of telemedicine implementation in thrombolytic use for acute ischemic stroke: the University of Pittsburgh Medical Center Telestroke Network Experience. I Stroke Cerebrovasc Dis 2013;22:527-31.

67 Müller-Barna P, Hubert GJ, Boy S, et al. TeleStroke units serving as a model of care in rural areas: 10-year experience of the TeleMedical project for integrative stroke care. Stroke 2014;45:2739-44.

68 Kazley AS, Wilkerson RC, Jauch E, et al. Access to Expert Stroke Care with Telemedicine: REACH MUSC. Front Neurol 2012:3:44.

69 Audebert HJ, Kukla C, Vatankhah B, et al. Comparison of tissue plasminogen activator administration management between Telestroke Network hospitals and academic stroke centers: the Telemedical Pilot Project for Integrative Stroke Care in Bavaria/Germany. Stroke 2006;37:1822-7. 
70 Goldstein LB, Matchar DB, Hoff-Lindquist J, et al. VA Stroke Study: neurologist care is associated with increased testing but improved outcomes. Neurology 2003;61:792-6.

71 Meyer BC, Raman R, Ernstrom K, et al. Assessment of long term outcomes for the STRokE DOC telemedicine trial (STRokE DOC-LTO). J Stroke Cerebrovasc Dis 2012;21:259-64

72 Zaidi SF, Jumma MA, Urra XN, et al. Telestroke-guided intravenous tissue-type plasminogen activator treatment achieves a similar clinical outcome as thrombolysis at a comprehensive stroke center. Stroke 2011;42:3291-3.

73 Mocco J, Fiorella D, Fargen KM, et al. Endovascular therapy for acute ischemic stroke is indicated and evidence based: a position statement. J Neurointerv Surg 2015;7:79-81.

74 Adeoye 0, Albright KC, Carr BG, et al. Geographic access to acute stroke care in the United States. Stroke 2014;45:3019-24.

75 Powers WJ, Derdeyn CP, Biller J, et al. 2015 AHA/ASA focused update of the 2013 guidelines for the early management of patients with acute ischemic stroke regarding endovascular treatment: a guideline for healthcare professionals from the American Heart Association/American Stroke Association. Stroke Published Online First: 29 Jun 2015. doi:10.1161/STR.0000000000000074
76 Heldner MR, Zubler C, Mattle HP, et al. National Institutes of Health Stroke Scale score and vessel occlusion in 2152 patients with acute ischemic stroke. Stroke 2013;44:1153-7.

77 Prabhakaran S, Ward E, John S, et al. Transfer delay is a major factor limiting the use of intra-arterial treatment in acute ischemic stroke. Stroke 2011:42:1626-30.

78 Pfefferkorn T, Holtmannspötter M, Schmidt C, et al. Drip, ship, and retrieve: cooperative recanalization therapy in acute basilar artery occlusion. Stroke 2010:41:722-6.

79 Pedragosa À, Alvarez-Sabín J, Rubiera M, et al. Impact of telemedicine on acute management of stroke patients undergoing endovascular procedures. Cerebrovasc Dis 2012;34:436-42.

80 Audebert HJ, Schultes K, Tietz V, et al. Long-term effects of specialized stroke care with telemedicine support in community hospitals on behalf of the Telemedical Project for Integrative Stroke Care (TEMPiS). Stroke 2009;40:902-8.

81 Joubert J, Joubert L, Reid C, et al. The positive effect of integrated care on depressive symptoms in stroke survivors. Cerebrovasc Dis 2008;26:199-205.

82 Lavallée $\mathrm{PC}$, Meseguer $\mathrm{E}$, Abboud $\mathrm{H}$, et al. A transient ischaemic attack clinic with round-the-clock access (SOS-TIA): feasibility and effects. Lancet Neurol 2007:6:953-60. 